\title{
Molecular environmental soil science at the interfaces in the Earth's critical zone
}

\author{
Jianming Xu • Caixian Tang • Jizheng He
}

Received: 25 March 2010 /Accepted: 31 March 2010/Published online: 23 April 2010

(C) Springer-Verlag 2010

The International Symposium of Molecular Environmental Soil Science at the Interfaces in the Earth's Critical Zone (ISMESS 2009) was successfully held during 10-14 October 2009 at Zhejiang University in Hangzhou, China. Two hundred sixty-six participants from 21 countries attended the symposium. The scientific program featured 2 plenary lecturers, 19 invited lecturers, 29 oral presentations, and 65 poster presentations.

The five main sessions were included in the symposium: (1) The role of mineral colloids in carbon turnover and sequestration and the impact on climate change; (2) Biogeochemical interfacial reactions and the transformation, transport, and fate of vital and toxic elements; (3) Anthropogenic organics, crop protection, and ecotoxicology; (4) Environmental nanoparticles: distribution, formation, transformation, structural, and surface chemistry and biogeochemical and ecological impacts; and (5) Environmental processes and ecosystem health (Xu and Huang 2009). Following the peer reviewing process, a selection of articles arising from the symposium is published in this issue of Journal of Soils and Sediments.

Responsible editor: Jianming $\mathrm{Xu}$

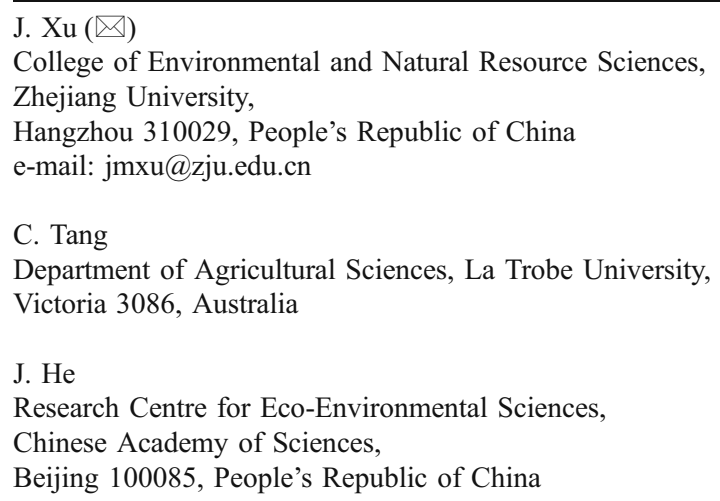

This symposium provided a forum for the interactions and communication of soil chemists, mineralogists, microbiologists, and physicists with allied scientists including pure chemists, biologists, environmental scientists, ecologists, and ecotoxicologists to address the current state-ofthe-art on "Molecular Environmental Soil Science". No doubt this symposium contributed to identification of gaps in knowledge and to future research directions and research on soil processes at the interfaces at the molecular level in the Earth's critical zone. It is significant to advance the frontiers of knowledge on biophysicochemical processes in soil and related environmental systems and their biogeochemical and ecological impacts and to promote education in this extremely important and challenging area of science for years to come.

The symposium was highly evaluated by all the participants, and major knowledge shared include the following: (1) The concept of the Earth's critical zone was highlighted, which is a system of coupled chemical, biological, physical, and geological processes operating together to support life at the Earth's surface. (2) Further efforts are required by scientists to cross-disciplines for the study of the interactive processes in the critical zone and their impact on the globe and humankind, ranging in scale from the environmental mineral-organism-humus-water-air interfaces. (3) Discussion on carbon turnover improved the understanding of the role of mineral colloids in carbon transformation, dynamics, and sequestration and their impact on climate change in the environment. (4) Microbes are involved in important natural biogeochemical processes relating to metal-mineral transformations, element cycling, bioweathering, biocorrosion, bioremediation, revegetation, phytoremediation, and containment of pollution in the Earth's critical zone. (5) The importance of nanoscience as a new frontier in soil science has been emphasized. The resolution is occurring for the study of 
processes of the key components of soils at the nanoscale. (6) The use of new advanced methods and approaches, such as synchrotron radiation and SIP-DNA, achieved an improved understanding for the environmental interfacial reaction processes and mechanisms in the Earth's critical zone.

This special issue identified and focused on emerging challenges for research that is predominantly crossdiscipline in nature. We hope that the articles presented in this special issue help to secure the appreciation of the relevance of molecular environmental soil science to the Earth's critical zone that is of utmost importance to sustain humankind. Finally, we would like to thank reviewers for their time and invaluable comments on improving the quality of the articles. Special thanks are also extended to the symposium sponsors: International Union of Soil Sciences, The International Union of Pure and Applied Chemistry, Organization for the Prohibition of Chemical Weapons, National Natural Science Foundation of China, Soil Science Society of China, Zhejiang University, and Zhejiang Provincial Key Laboratory of Subtropical Soil and Plant Nutrition.

\section{Reference}

Xu JM, Huang PM (eds) (2009) Molecular environmental soil science at the interfaces in the earth's critical zone. Zhejiang University Press \& Springer, Hangzhou 